\title{
The Effect of Moderate-Fidelity Mannequin VS Peer Simulated Patient on Nursing Students Experience and Level of Competency
}

\author{
Samah Saad Salem ${ }^{1}$ \& Lerma C. Cabaldo ${ }^{2}$.
}

1. Lecturer, Medical-Surgical Nursing Department, Faculty of Nursing, Cairo University, Cairo, Egypt. Assistant Professor, College of Nursing, King Saud bin Abdulaziz University for Health Sciences, Riyadh ,Saudi Arabia. King Abdullah International Medical Research Center, Riyadh, Saudi Arabia.

2. Teaching Assistant, Maternal and Child Care Nursing, College of Nursing, King Saud bin Abdulaziz University for Health Sciences, Riyadh ,Saudi Arabia. King Abdullah International Medical Research Center, Riyadh, Saudi Arabia.

\begin{abstract}
Background: Simulation in nursing has been the trend as one of the teaching modalities during laboratory session. Contemporaneous, the use of mannequin and peer simulated patient serves as a medium to depict patient in providing necessary competencies in the realm of nursing mainly in Health Assessment course. Aim: To investigate the effect of moderate-fidelity mannequin vs peer simulated patient on nursing students experience and level of competency. Method: 80 nursing students enrolled in Health Assessment course at King Saud bin Abdulaziz University for Health Science were recruited in the current study; data was collected by questionnaire and competency checklist. Results: The study results revealed that there was a statically significance difference regarding the total experience and total competency mean scores among the study and control groups $(\mathrm{t}=10.039, \mathrm{P}=$ $.000 \& \mathrm{t}=3.791, \mathrm{P}=.000$ respectively) towards the use of Peer Simulated Patient. Conclusion and Recommendation: Nowadays many nursing schools have started to utilize simulation-based nursing teaching and learning, however, simulation-based learning should be applied blended with the peer simulation in teaching to improve the undergraduate nursing students' clinical experience and assessment skills.
\end{abstract}

Keywords: Simulation, Moderate Fidelity Mannequin, Peer-Simulated Patient, Students Experience \& Level of Competency

\section{Introduction}

Simulation has an extensive and varied past in numerous diverse fields. (Aebersold, 2016). Simulation is defined as mock practice that characterizes its practice completed for a time. In a systematic review, simulation nursing was described as a motivating intervention that offers additional insights than those that assessed or tested effectiveness. (Cleaver, et al. 2021). Simulation implements different types of frameworks to facilitate learning. It works well for beginners and advanced learners (Chernikova, et al., 2020). The simulationbased medical education enables the student to absorb and master technical, non-technical skills in realistic situations. (Bogár, et al., 2020) Simulation has significant role in medical training. It supports standardization of education and guarantee the quality and comparability through extending education environment (Everett, et al., 2019).

In nursing education, simulation has become an integral part and defined as "program or event replicating the clinical exercise using scenarios, highfidelity mannequins, medium-fidelity mannequin, standardized patients, role playing, skills station, and computer-based critical thinking simulations. (Sofer, 2018) The American Association of Colleges of Nursing (AACN) and the Commission on Collegiate Nursing Education (CNE) has a viewpoint that the most significant section of clinical teaching is actual patient care experiences. The methodologies of simulation in nursing education are significant in relations to reducing student's anxiety in harmless lifelike environment for the enhancement of their knowledge and skills in clinical applications. (Doğru \& Aydın, 2020).

Accordingly, successive simulations and students experience may lead to student's satisfaction and selfconfidence. (Zapko et al., 2018). For the undergraduate nursing programs in the United States, simulation is fast becoming a normative teaching for a portion of clinical time. (Lubbers \& Rossman , 2017).

Simulated patient (SPs) is frequently use for students training in communication skills. The advantage of using SPs is the chance to deliver standardized method by which participants and students may reveal and improve communications skills. (MacLean, et al., 2018) 
On the aspect of training based, clinical simulation is an active technique of education in nursing. However, no strong evidence if it is adequate to use-or medium fidelity simulation. (Alconero-Camarero, et al. 2021). Furthermore, a study suggested that the use of combined training methods (simulations and critical thinking strategies) increase students' practical knowledge in a safe and controlled environment. (Zarifsanaiey, Mitra , \& Saadat, 2016). As educational approach, the integration of simulation in the curriculum is feasible in low-resource situations through the use of low-and medium fidelity simulation. It promotes extra learning experiences that leads to the improvement of knowledge, skills and attitudes. (Martins et al., 2018)

Peer simulation appears to be an effective technique for offering training in clinical skills for health care students. It recognized obstacles to simulation-based education application. Similar to peer instruction, the flipping of roles throughout peer simulation offers typical assistance to students. It has a constructive effect to student's communication, confidence, and self-efficacy. (Dalwood, et al., 2020). Likewise, in a pragmatic observational study, peer simulation might be a viable alternative to simulation-base education with simulated patients. (Pritchard, et al., 2020)

On the other hand, peer-learning method has a positive effect on the learning outcomes. Besides clinical site peer, learning is greater than laboratory. (Choi, Kim, Park, Lim, \& Kim, 2021). Moreover, in higher education, peer instruction provides a teaching method that increases learning. This is generally use in the variety of courses and countries to have an interaction during lectures. (Balta, et al., 2017). Likewise, it was emphasized in peer mentorship it can be applied largely for teaching and learning within the health care learning settings. (Andersen \& Watkins, 2018) \& (MjangerEide, Johansson, \& Eide, 2020).

In the current years, peer learning has shown to be factual learning intervention for students in clinical health science and it helps in acquiring information and skills through active support among equal peers and empowers students to practice their communication skills, cooperation, reflection, and independence. (Nakayama, et al., 2021), (Pålsson, et al., 2017) \& (Carey, Kent, \& Latour, 2018).

The students' experience of the simulation-based training remains as continuing and conscious learning outcomes throughout the completion of student's clinical practice. However, the organization of simulation-based training and its application in the curriculum are vital for the learning outcomes of students' experiences to bridge knowledge to clinical practice. (Hustad, et al., 2019), (Cantrell, et al., 2017) \& (Kumar, et al., 2017). Within the program of assessment for core competencies, simulation has a role in higher-stakes assessment that is rarely demonstrable then the management cannot be delegated to a trainee for assessing the clinical environment (Dupre \& Naik, 2021) \& (Bond, et al., 2017). One article noted that simulation-based education guarantees provider competency within the health care system. (Griswold, et al., 2018).

\section{Significant of the study}

In an integrative review of Inter professional (IP) competencies that delivers a present state of information on the effectiveness of inter professional simulation in increasing learning and competencies of nursing student. Through interpersonal simulation experience, nurse academe can satisfactorily prepare nursing students for upcoming combined practice. (Labrague et al., 2018). Nevertheless, thru patient safety as a priority; there is still a challenge in nursing education wheter to change from task-based competences to higher-level competencies. (Badowski \& Oosterhouse, 2017). There was no studies investigated which method has better impact on Students' Experience and Level of Competency neither in Saudi Arabia nor any other Arab country. So, the current study aimed to investigate the impact of moderate-fidelity mannequin vs peer simulated patient on nursing students' experience and level of competency in health assessment course.

Aim of the study

The aim of the current study was to investigate the effect of moderate-fidelity mannequin vs peer simulated patient on nursing students experience and level of competency.

\section{Research Hypotheses:}

H1- Nursing students who will practice using moderate-fidelity mannequin will have better experience than those who will practice using peer simulated patient.

H2- Nursing students who will practice using moderate-fidelity mannequin will have higher level of competency than those who will practice using peer simulated patient.

Research question

What are the differences of the impact of moderatefidelity mannequin vs peer simulated patient on nursing students' experience and level of competency in health assessment course?

\section{Materials and Methods:}

\section{Study Design:}

A quasi-experimental research design was used to investigate the impact of moderate-fidelity mannequin vs peer simulated patient on nursing students' experience and level of competency in health assessment course. 


\section{Study Setting:}

The current study was conducted at College of Nursing, King Saud bin Abdulaziz University for Health Sciences, Riyadh, Saudi Arabia. The health assessment course is one of the basic courses that provided to level 5 students. The study will principally conducted in the college's skills laboratory.

\section{Study Subjects:}

A total number of 80 nursing students enrolled in health assessment course used a convenient sampling technique then the students were recruited in either control or study group randomly (40 students in each) during fall and spring semesters, annual year 20162017 was recruited in the current study according to the following inclusion criteria; Saudi, female, willing to voluntarily participate in the study and enrolled in health assessment course for the first time.

\section{Sample Size:}

Using the $G$ power software with a paired sample ttest, a p-value of 0.05 , a power of 0.80 and effect size of 0.7 , the calculated sample size was 68 subjects. The number was increased to 80 to ensure representativeness

\section{Data collection instrument:}

Two tools were used in the current study developed by researchers after reviwed extensive relevant literature

Tool I: structured self-Administered questionnaire: it included two parts;

Part I: its purpose to assess personal characterisyics of nursing students such as; age, nationality, and marital status.

Part II: Related to the participants experience toward moderate fidelity mannequin/peer simulated patient. This part contained 10 questions and has been rated using the 4 point Likert scale ranging from 1 to 4 . The lowest possible score was 10 while the highest possible one was 40 , the higher the score the more positive the experience. Also the satisfaction level of the participants experience classified to 4 amin categories from 0 to 10 dissatisfied, from 11 to 20 had low satisfactory experience, from 21 to 30 had moderately satisfactory experience and from 31 to 40 highly satisfactory experience

Content and item valaididty were tested by three experts in nursing field and the reliability of the second part of the questionnaire was assessed using Cronbach Alpha and was 0.74.

Tool II: The competency checklists was the thorax and lung assessment checklist and cardiovascular assessment checklist adopted from (Wilkinson \& Leuven 2017) they were valid and reliable tools and present in the textbook for free uses. The competency checklist measured the student's competence level while using the different four health assessment techniques namely; inspection, palpation, percussion and auscultation techniques. The posible total score for both checklist was 10 score, the higher the score the the higher the competency level.

A pilot study was applied on 10 students to assess the clarity and applicability of the study tool, they were included in the study because no major changes were required.

\section{Data collection:}

After explaining the purpose of the study to the participants and obtaining their verbal agreement, each participant received and signed the hard copy of the King Abdullah International Medical Research Center (KAIMRC) consent form. Then the researcher started to used the moderate-fidelity mannequin during the lab session for the study group (40 participants) while the control group (40 participants) proceeding with the peer simulated patient which is routinely teaching method used in health assessment course laboratory session. Two moderate-fidelity mannequins have been used for respiratory and cardiovascular assessment. The researchers exposed all the students to the moderate-fidelity mannequins for fairness between all students. Then thereafter, the researchers introduced the study instruments to assess the students' experience. To assess the competency level of the students, the researchers used the assessment checklist. The marks has been calculated based on the total score obtained by the students divided by the total score of the assessment checklist. The researchers presented only the mean score of the Thorax and Lungs Assessment and Cardiovascular Assessment. The data collection has been take place during the period fall and spring semesters of the annual year 2016-2017.

Data Management and Analysis Plan:

All data was entered and analyzed using the Statistical Package for the Social Science (SPSS, version 21). Statistical significance will be set at $\mathrm{p}=<$ 0.05 ; and the the confidence interval at $95 \%$. Descriptive statistical analysis as percentage, frequency distribution, mean, and median will be used to describe the research sample and participant responses to questionnaire items. Chi-square test will be used as a bivariate analyses between dependent and independent variables. To measure the correlations between some selected variables the product-moment correlation coefficients will be used. paired sample t-test to test the difference between two independent means (two groups).

\section{Ethical Considerations:}

The research proposal was approved by the Research Unit - College of Nursing, King Saud bin Abdulaziz University for Health Sciences, and Institutional Review Board (IRB) in King Abdullah International Medical Research Canter (KAIMRC), National Guard 
Health Affairs, Riyadh, Saudi Arabia with IRB \# (RC17/1504/17). Written informed consent was obtained from each participant prior starting the data collection. Each participant was informed about the purpose of the study, participant's anonymity was kept as well as data was kept highly confidential and secured and each participant has the right to withdraw from the research or refuse to share without any affection in his or her marks. After completion of the assessment tools and student evaluation of competencies, the researchers were, expose all the students to the moderate-fidelity mannequins for fairness between all students.

\section{Results:}

Regarding the frequency distribution of the demographic characteristics of the participants. the mean age of the participants was $20.0625 \pm 4.34$ and all of them were Saudi females.

Table (1): Frequency distribution of the participants experience toward moderate fidelity mannequin/peer simulated patient in Health Assessment Course ( $N=80)$.

\begin{tabular}{|c|c|c|c|c|c|c|c|c|c|c|c|c|c|c|c|c|c|}
\hline & \multirow{3}{*}{ Variables } & \multicolumn{8}{|c|}{$\begin{array}{l}\text { Moderate Fidelity Mannequin } \\
(\mathrm{N}=40)\end{array}$} & \multicolumn{8}{|c|}{$\begin{array}{l}\text { Peer Simulated Patient } \\
\qquad(N=40)\end{array}$} \\
\hline & & \multicolumn{2}{|c|}{$\begin{array}{c}\text { No/Not } \\
\text { (1) }\end{array}$} & \multicolumn{2}{|c|}{$\begin{array}{c}\text { Low } \\
(2)\end{array}$} & \multicolumn{2}{|c|}{\begin{tabular}{|c|} 
Moderate \\
(3)
\end{tabular}} & \multicolumn{2}{|c|}{$\begin{array}{c}\text { High } \\
\text { (4) }\end{array}$} & \multicolumn{2}{|c|}{$\begin{array}{l}\text { No/Not } \\
\text { (1) }\end{array}$} & \multicolumn{2}{|c|}{$\begin{array}{c}\text { Low } \\
(2)\end{array}$} & \multicolumn{2}{|c|}{\begin{tabular}{|l} 
Moderate \\
(3)
\end{tabular}} & \multicolumn{2}{|c|}{$\begin{array}{l}\text { High } \\
\text { (4) }\end{array}$} \\
\hline & & \# & $\%$ & \# & $\%$ & $\#$ & $\%$ & \# & $\%$ & \# & $\%$ & $\#$ & $\%$ & \# & $\%$ & $\#$ & $\%$ \\
\hline 1. & Satisfaction & 16 & $40 \%$ & 1 & $2.5 \%$ & 22 & $55 \%$ & 1 & $2.5 \%$ & 0 & $0 \%$ & 2 & $5 \%$ & 31 & $77.5 \%$ & 7 & $17.5 \%$ \\
\hline 2. & Confidence & 7 & 17.5 & 0 & $0 \%$ & 25 & $62 . \%$ & 8 & $20 \%$ & 0 & $0 \%$ & 2 & $5 \%$ & 18 & $45 \%$ & 20 & $50 \%$ \\
\hline 3. & Comfort & 7 & $17.5 \%$ & 2 & $5 \%$ & 24 & $60 \%$ & 7 & $17.5 \%$ & 0 & $0 \%$ & 1 & $2.5 \%$ & 21 & $52.5 \%$ & 18 & $45 \%$ \\
\hline 4. & Familiarity & 4 & $10 \%$ & 11 & $27.5 \%$ & 23 & $57.5 \%$ & 2 & $5 \%$ & 0 & $0 \%$ & 2 & $5 \%$ & 30 & $75 \%$ & 8 & $20 \%$ \\
\hline & $\begin{array}{l}\text { Critical thinking } \\
\text { enhancement }\end{array}$ & 4 & $10 \%$ & 9 & $22.5 \%$ & 17 & $42.5 \%$ & 10 & $25 \%$ & 0 & $0 \%$ & 0 & $0 \%$ & 24 & $60 \%$ & 16 & $40 \%$ \\
\hline 6. & \begin{tabular}{|l|} 
Refining \\
Memorization.
\end{tabular} & 8 & $20 \%$ & 5 & $12.5 \%$ & 18 & $45 \%$ & 9 & $22.5 \%$ & 0 & $0 \%$ & 0 & $0 \%$ & 25 & $62.5 \%$ & 15 & $37.5 \%$ \\
\hline 7. & $\begin{array}{l}\text { Displays } \\
\text { abnormality }\end{array}$ & 7 & $17.5 \%$ & 4 & $10 \%$ & 17 & $42.5 \%$ & 12 & $30 \%$ & 2 & $5 \%$ & 12 & $30 \%$ & 16 & $40 \%$ & 10 & $25 \%$ \\
\hline 8. & $\begin{array}{l}\text { Applicable for all } \\
\text { steps. }\end{array}$ & 15 & $37.5 \%$ & 14 & $35 \%$ & 11 & $27.5 \%$ & 0 & $0 \%$ & 0 & $0 \%$ & 2 & $5 \%$ & 24 & $60 \%$ & 14 & $35 \%$ \\
\hline 9. & Realistic. & 23 & $57.5 \%$ & 8 & $20 \%$ & 9 & $22.5 \%$ & 0 & $0 \%$ & 0 & $0 \%$ & 0 & $0 \%$ & 25 & $62.5 \%$ & 15 & $37.5 \%$ \\
\hline 10. & $\begin{array}{l}\text { Helpful in clinical } \\
\text { application. }\end{array}$ & 6 & $15 \%$ & 7 & $17.5 \%$ & 14 & $35 \%$ & 13 & $32.5 \%$ & 0 & $0 \%$ & 2 & $5 \%$ & 16 & $40 \%$ & 22 & $55 \%$ \\
\hline & $\begin{array}{l}\text { otal experience } \\
\text { mean score } \\
\text { Mean }+ \text { SD }\end{array}$ & \multicolumn{8}{|c|}{$25.18 \pm 4.01$} & \multicolumn{8}{|c|}{$32.95 \pm 2.810$} \\
\hline $\begin{array}{l}\text { In } \\
\text { P }\end{array}$ & $\begin{array}{l}\text { dependent } \mathrm{t} \text { test } \\
\text { value }\end{array}$ & \multicolumn{16}{|c|}{$\begin{array}{c}10.039 \\
.000\end{array}$} \\
\hline
\end{tabular}

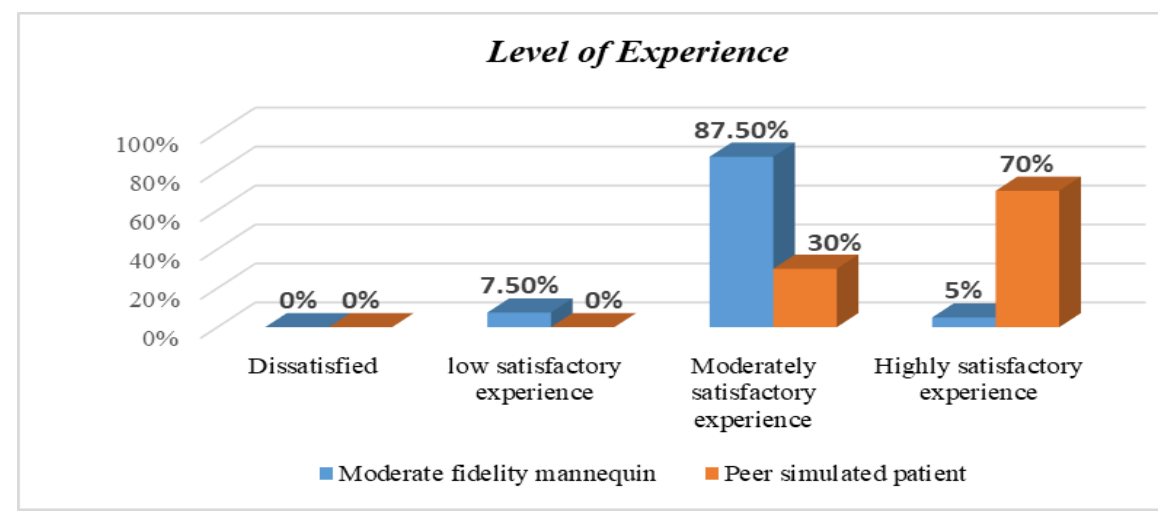

Figure (1): Level of Experience toward Moderate Fidelity Mannequin/Peer Simulated Patient in Health Assessment course (N=80) 
Table (2): Level of Competency toward Moderate Fidelity Mannequin/Peer Simulated Patient in Health Assessment course among the participants. $(\mathrm{N}=80)$

\begin{tabular}{|l|c|c|}
\hline \multicolumn{1}{|c|}{ Total competencies mean score. } & Moderate Fidelity Mannequin & Peer Simulated Patient \\
\cline { 2 - 3 } Mean $\underline{\mathbf{S D}}$ & $\mathbf{9 . 1 8 + \mathbf { 1 . 1 }}$ & $\mathbf{9 . 8 7 + \mathbf { 0 . 3 4 }}$ \\
\hline Independent $\mathbf{t}$ test & \multicolumn{2}{|c|}{3.791} \\
P value & \multicolumn{2}{|c|}{.000} \\
\hline
\end{tabular}

Table (1): Showed the frequency distribution of the participants experience toward moderate fidelity mannequin/peer simulated patient in Health Assessment Course. Participants were moderately satisfied with use of moderate fidelity mannequin $(55 \%)$ and peer simulated patient $(77.5 \%) .62 .5 \%$ of the participants were moderate confident in using the moderate fidelity mannequin, while $50 \%$ of the participants showed that they are highly confident in peer simulated patient. $60 \%$ of the participant is moderately comfortable in using moderate fidelity mannequin while $52.5 \%$ of the participant from peer simulated patient illustrated their moderately comfort in peer simulated patient. Both participants were moderately familiar from the moderate fidelity mannequin (57.5\%) and peer simulated patient (75\%). Besides, both groups, Moderate fidelity mannequin $42.5 \%$ and peer simulated patient $60 \%$ shows that they have moderately enhancement of critical thinking. In refining the memorization, both groups showed moderately result, $45 \%$ responded for moderate fidelity mannequin and $62.5 \%$ responded for peer simulated patient. Moreover, on the display of abnormality both groups rated moderately experience, moderately fidelity mannequin has $42.5 \%$ while peer simulated patient has $40 \%$ result. Evidently, in the application for all steps, moderate fidelity mannequin has a lower respond $37.5 \%$ as not applicable for all the steps comparing to peer simulated patient that has moderately responds of $60 \%$. Likewise, for realistic moderate fidelity mannequin got the lowest response of $57.5 \%$ comparing to $62.5 \%$ of moderately realistic to peer simulated patient. Lastly, both groups responded that moderate fidelity mannequin $(32.5 \%)$ and peer simulated patient $(55 \%)$ is highly helpful in their clinical application. Regarding the total experience mean score among the study and control group as shown it revealed that there was a statically significance difference between both groups $(\mathrm{t}=$ $10.039 \& \mathrm{P}=.000$ ) towards the use of Peer Simulated Patient.

Figure (1): Showed that none of both groups were dissatisfied $(0 \%)$, for who reported the low satisfactory experience $7.5 \%$ was from the study group and $0 \%$ from the control group, for who reported the moderately satisfactory experience $87.5 \%$ was from the study group while only $30 \%$ from the control group, as regarding the highly satisfactory experience only 5\% was from the study group while $70 \%$ was from the control group.

Table (2): Showed the Level of Competency toward Moderate Fidelity Mannequin/Peer Simulated Patient in Health Assessment course among the participants and revealed total competency mean score, mean \pm $\mathrm{SD}$, of the participants who used the Moderate fidelity mannequin versus the participants who used the Peer simulated patient were $(9.18 \pm 1.1$ and $9.87 \pm$ 0.34 respectively). It was also revealed that there was a statically significance difference between both groups $(\mathrm{t}=3.791 \& \mathrm{P}=.000)$ towards the use of Peer Simulated Patient.

\section{Discussion:}

The aim of the current study was to investigate the effect of moderate-fidelity mannequin vs peer simulated patient on nursing students experience and level of competency.

\section{Characteristics of the participants}

The study results showed that participants had an average age group of 20; all of them were Saudi female. In the medical setting, previous studies found that female health care givers present further empathy with patients than their male counterparts do. However, (A.Strekalova et al., 2019) reported that the level of emphatic responses showed no differences between male and female students. Also, College of Nursing, King Saud bin Abdulaziz University for Health Sciences, Riyadh, Saudi Arabia accepted only females for enrollment in nursing program until this moment.

\section{Experience toward moderate fidelity mannequin/peer simulated patient}

One of the purposes of this study is to evaluate the impact of moderate-fidelity mannequin on nursing students' experience in health assessment course. The comparisons made between moderate fidelity and peer simulations to investigate the educational impact. The study results showed that out of ten (10) variables in evaluating students experience towards moderate fidelity mannequin: satisfied, confidence, comfortable and familiar in using moderate fidelity mannequin received higher percentage. The result is congruent to (Handeland et al., 2021) that mannequin offers improvement of professional identity, development a caring communication skill 
that increases sense of realism and experience what it means to behave as nurses. Interestingly, parallel to article (Aarkrog, 2019) high degree of fidelity simulation involves students' knowledge that enables them to try out practical skills. Although, peculiar result of (Basak et al., 2016) perceived that high fidelity mannequins brings to be higher in contrast to student's perceptions of experiences using low fidelity mannequins. This is in contrary to the study of (Munshi, Lababidi, \& Alyousef, 2015) stating that High fidelity is not always superior to lower fidelity because this is dependent on the task and the learners. Besides (Tutticci et al., 2016) reported that a high level of satisfaction with high fidelity simulation experience, agreed with, and valued the educational practices associated with simulation.

Whereas the current results showed that participants were more satisfied in dealing with peer-simulated patient rather than the mannequin. Out of ten variables, six items received greater percentage; Students are more satisfied, familiar, improves memory, realistic and enhances critical thinking and peer simulated is applicable for all steps. Parallel to the quantitative and qualitative study of (Brown \& Rode, 2018), students expressed their satisfaction and confidence in mastering the necessary skills. However, the meta-analysis effect of (Balta et al., 2017) during the observation of both informal and cultural variables. It was reported that peer instruction has a greater impact on learning compared to traditional techniques. Corresponding to the experience of nursing students with standardized patients in simulation, (Ha, 2018) obtained three viewpoints: 1) SPs are helpful for patient care (SPs patient-centered view), 2) SPs parts are important for nursing student learning (SPs roles-centered view) and 3) SPs can motivates competency of nursing students (student-centered view).

\section{Level of Competency toward Moderate Fidelity Mannequin/Peer Simulated Patient}

Regarding the level of competency toward moderate fidelity mannequin/peer simulated patient in health assessment course among the participants. This study reveals that the level of competency among students has no significant difference when utilizing moderate fidelity mannequin or peer-simulated patient; although the result showed slightly greater than those who used the moderate fidelity mannequin. This is in contrary to the comparative group design of (Lee, et al. 2019) where student's practicum grades are collected to examine their clinical performance. They found positive significant differences on five competencies in the test group. There was a significant difference on the professional knowledge and nursing process subscale, nonetheless no significant differences found in the patient safety, communication, and attitude of reflection subscales. This is in matching positive, significant differences in five competencies in the test group. Overall, no significant findings in clinical performance between two groups. Although (L.P \& VanHorn , 2019) stated in a comprehensive literature synthesis that there was a significant need for future research involving validated tools and process for the evaluation of students' competencies in the clinical setting.

Level of Experience toward Moderate Fidelity Mannequin/Peer Simulated Patient in Health Assessment course

Interestingly, student's level of experience toward the use of moderate fidelity mannequin vs peer simulated patients in health assessment course as shown in figure 1, reveals that none of the groups were dissatisfied. Besides, students who operates moderate fidelity mannequin were $87.50 \%$ moderately satisfied while students experience with their peer as the simulated patient receives $70 \%$ highly satisfactory. Apparently, realism takes issue in this simulation. Although in the study of MacLean, et al., (2019) the participants had difference perception in the realism and they attendance were not affected by the communication-based learning interventions.

Moreover, in the independent sample t test of two students' groups (Johnson, et al., 2020) the result reported greater satisfaction, and improved communication for those who completed the simulation with SP. Furthermore, there was no statistical difference between the two groups in selfconfidence. Additionally, in the systematic review of (Labragu, et al., 2019) result shows improvement of student's self-confidence after a clinical simulationbased activity, which significantly increase their satisfaction, knowledge, psychomotor skills, assessment and communication.

Correlation between the experience and competency level among the study and control group.

In this study, we examined the correlation of simulation experience to competency. Although, evaluation of clinical competence is multifaceted, and conclusion based. Truthful and strong assessment process needs favorable proof of validity, mainly when decisions are considered high stake. (Tavares, et al., 2018). The sample of 80 students was satisfied both in the used of moderate fidelity mannequin and peer simulated patient. Nevertheless, the study results signified that there was statistically difference in the total experience mean score and total competency mean score between the two groups towards the use of Peer Simulated Patient.

In connotation with the quasi-experimental pretest/posttest design (Kirkpatrick, et al., 2019) in 
linking acquired clinical competency among students, the report was highly experienced in pre-simulation. Although pretest self-awareness and knowledge were higher in students with more than two types of experience, there were no significant differences in the outcome of groups for pre-or post-simulation. Post simulation outcomes were higher and found to have moderate correlations. However, in the randomized controlled trial of (Padilha, et al., 2019) the experimental groups made significant improvement in the knowledge after intervention and 2 months later. It signifies that there was a higher level of learning satisfaction.

\section{Conclusions:}

Nowadays many nursing schools have started to utilize simulation-based nursing teaching and learning, however, simulation-based learning should be applied blended with the peer simulation in teaching the health assessment course to improve the undergraduate nursing students' clinical experience and assessment skills.

\section{Recommendations:}

The researchers recommended further studies to be done to explore the Impact of Moderate-Fidelity Mannequin VS Peer simulated Patient on Nursing Students' Experience and Level of Competency in Health Assessment Course. Further studies are also required to explore the pros and cons for each taching and learning strategies. Replication of this study is also recommended taking into consideration the stated limitation.

\section{Limitations:}

The limitation of this study should be acknowledged. First, the study was carried out in to two-simulation approach, thus the result cannot be inferred to the students with either moderate fidelity or peersimulated mannequin. The said approach may affect the particularities of each setting. Second, the student's level of learnings that gives impact on their level of competence. In this regard, future research should consider the simulation approach to isolate the effect of the differences in the level of satisfaction and considers the learning process in more complete way encourage.

\section{Relevance to Clinical Practice:}

This study provided important findings regarding the use of fidelity mannequins and peer-simulated patient in teaching the health assessment course to improve the undergraduate nursing student clinical performance. It has an impact in understanding the clinical and academic uses of simulation.

\section{Authors' contributions}

All authors were responsible for study conception and design, data collection, analysis, and interpretation.
All authors critically read and approved the final manuscript for important intellectual content.

\section{Acknowledgment}

The authors would like to express their gratitude to all participants who gave their time to participate in this study.

Disclosure of interest

No conflict of interest to declare.

\section{References}

- A.Handeland, J., Prinz, A., Ekra, E., \& Fossum, M. (2021): The role of manikins in nursing students' learning: A systematic review and thematic metasynthesis. Nurse Education Today.

- A.Strekalova, Y., Kong, S., Kleinheksel, A., \& Gerstenfeld, A. (2019): Gender differences in the expression and cognition of empathy among nursing students: An educational assessment study. Nurse Education Today, 1-6.

- Aebersold, M. (2016): The History of Simulation and Its Impact on the Future. AACN Advanced Critical Care, 56-61.

- Alconero-Camarero, A., Cobo, C., Piris, M., Gómez, S., \& López, J. (2021): Nursing Students' Satisfaction: A Comparison between Medium- and High-Fidelity Simulation Training. International Journal of Environmental Research and Public Health.

- Badowski, D., \& Oosterhouse, K. (2017): Impact of a Simulated Clinical Day With Peer Coaching and Deliberate Practice: Promoting a Culture of Safety. Nursing Education Perspectives, 93-95.

- Basak, T., Unver, V., Moss, J., Watts, P., \& Gaioso, V. (2016): Beginning and advanced students' perceptions of the use of low- and highfidelity mannequins in nursing simulation. Nurse Education Today, 37-43.

- Bogár, P., Tóth, L., Rendeki, S., Mátyus, L., Németh, N., Boros, M., \& Maróti , P. (2020): The present and the future of medical simulation education in Hungary. Orvosi Hetilap, 1078-1087.

- Bond, W., Gonzales, H., Funk, A., Fehr, L., McGarvey, J., Svendsen, J., \& Sawicki, R. (2017): Deliberate Practice with Standardized Patient Actors and the Development of Formative Feedback for Advance Care Planning Facilitators. Journal of Palliative Medicine, 631-637.

- Brown, K., \& Rode, J. (2018): Leadership Development Through Peer-Facilitated Simulation in Nursing Education. The Journal of Nursing Education, 53-57.

- Chernikova, O., Heitzmann, N., Stadler, M., Holzberger, D., Seidel, T., \& Fischer, F. (2020): Simulation-Based Learning in Higher Education: A Meta-Analysis. Review of Education Research, 143. 
- Choi, J., Kim, O., Park, S., Lim, H., \& Kim, J.H. (2021): Research on the effective of peer instruction and students' involvement. Asia-Pacific of Multimedia Services Convergent with Art. Clinical Simualtion Nursing, 92-101.

- Cleaver, K., Essex, R., Malamateniou, C., Shekede, H., \& Weldon, S. (2021): A Systematic Scoping Review and Textual Narrative Synthesis of Undergraduate Pediatric Nursing Simulations: What, Why, and How? Clinical Simulation in Nursing, 10-31.

- Dalwood, N., Bowles, K.-A., Williams, C., Morgan, P., Pritchard, S., \& Blackstock, F. (2020): Students as patients: A systematic review of peer simulation in health care professional education. MEDICAL EDUCATION IN REVIEW, 387-399.

- Doğru, B., \& Aydın, L. (2020): The effects of training with simulation on knowledge, skill and anxiety levels of the nursing students in terms of cardiac auscultation: A randomized controlled study. Nurse Education Today.

- Dupre, J., \& Naik, V. (2021): The role of simulation in high-stakes assessment. BJA Education, 148-153.

- Everett, E. , Forstein, D., Bliss, S., Buery-Joyner, S., Craig, L., Graziano, S. \& Page-Ramsey, S. (2019): To the Point: The expanding role of simulation in obstetrics and gynecology medical student education. American Journal of Obstetric and Gynecology, 129-141.

- Griswold, S., Fralliccardi, A., Boulet, J., Moadel, T., Franzen, D., Auerbach, M., \& Gordon, J. (2018): Simulation-based Education to Ensure Provider Competency Within the Health Care System. Academic Emergency Medicine (pp. 168176). Orlando, Florida: Society for Academic Emergency Medicine.

- Ha, E.-H. (2018): Experience of nursing students with standardized patients in simulation-based learning: Q-methodology study. Nurse Education Today, 123-129.

- Hustad, J., Johannesen, B., Fossum, M., \& Hovland, O. (2019): Nursing students' transfer of learning outcomes from simulation-based training to clinical practice: a focus-group study. BMC Nursing.

- Johnson, K., Scott, A., \& Franks, L. (2020): Impact of Standardized Patients on First Semester Nursing Students Self-Confidence, Satisfaction, and Communication in a Simulated Clinical Case. SAGE Open Nursing.

- Kirkpatrick, A., Cantrell, M., \& Smeltzer, S. (2019): Relationships among nursing student palliative care knowledge, experience, self- awareness, and performance: An end-of-life simulation study. Nurse Education Today, 23-30.

- Kumar, A., Nestel, D., East, C., Hay, M., Lichtwark, I., McLelland , G. \& Wallace, E. (2017): Embedding assessment in a simulation skills training program for medical and midwifery students: A pre- and post-intervention evaluation. Australian and New Zealand Journal of Obstetrcis and Gynaecology, 40-46.

- L.P, L., \& VanHorn, R. (2019): The state of the science on clinical evaluation in nursing education. The state of the science on clinical evaluation in nursing education.

- Labrague, L., McEnroe-Petitte, D., Bowling , A., Nwafor, C., \& Tsaras, K. (2019):. High-fidelity simulation and nursing students' anxiety and self-confidence: A systematic review. Nursing Forum, 358-368.

- Lee, B.-O., Liang, H.-F., Chu, T.-P., \& Hung, C.C. (2019): Effects of simulation-based learning on nursing student competences and clinical performance. Nurse Education in Practice.

- Lubbers, J., \& Rossman, C. (2017): Satisfaction and self-confidence with nursing clinical simulation: Novice learners, medium-fidelity, and community settings. Nurse Education Today, 140144.

- MacLean, S., Geddes, F., Kelly, M., \& Phillip, D. (2019): Realism and Presence in Simulation: Nursing Student Perceptions and Learning Outcomes. The Journal of Nursing Education, 330338.

- Martins, J., Baptista, R., Coutinho, V., Fernandes, M., \& Fernandes, A. (2018): Simulation in Nursing and Midwirery Education. World Health Organization Regional Office for Europe.

- Munshi, F., Lababidi, H., \& Alyousef, S. (2015): Low- versus high-fidelity simulations in teaching and assessing clinical skills. Journal of Taibah University Medical Sciences, 12-15.

- Padilha, J., Machado, P., Ribeiro, A., Ramos, J., \& Costa, P. (2019): Correction: Clinical Virtual Simulation in Nursing Education: Randomized Controlled Trial. Journal of Medical Internet Research.

- Pritchard, S., Keating, J., Nestel, D., \& Blackstock, F. (2020): Physiotherapy students can be educated to portray realistic patient roles in simulation: a pragmatic observational study. BMC Medical Education, 20:471.

- Sofer, D. (2018): The Value of Simulation in Nursing Education. American Journal of Nursing, $17-18$ 
- Tavares, W., Brydges, R., Myre, P., Prpic, J., Turner, L., Yelle, R., \& Huiskamp, M. (2018): Applying Kane's validity framework to a simulation based assessment of clinical competence. Advances in Health Sciences Education: Theory and Practice, 323-338.

- Tutticci, N., Coyer, F., Lewis, P., \& Ryan, M. (2016): High-Fidelity Simulation: Descriptive Analysis of Student Learning Styles. Clinical Simulation in Nursing.

- Zapko, K., Ferranto, M., Blasiman, R., \& Shelestak, D. (2018): Evaluating best educational practices, student satisfaction, and self-confidence in simulation: A descriptive study. Nurse Education Today, 28-34.

- Zarifsanaiey, N., Mitra , A., \& Saadat, F. (2016): A comparison of educational strategies for the acquisition of nursing student's performance and critical thinking: simulation-based training vs. training (simulation and critical thinking strategies). BMC Medical Education.

- Zulkosky, K., White, K., \& Price, A. (2016): Effect of Simulation Role on Clinical DecisionMaking Accuracy. Clincial Simulation in Nursing, 98-106. 